\title{
Chloroquine Autophagic Inhibition Rebalances Th17/Treg-Mediated Immunity and Ameliorates Systemic Lupus Erythematosus
}

\author{
Ning An Yanwen Chen Chao Wang Chen Yang Zhi-hong Wu Jing Xue \\ Lin Ye Shujun Wang Hua-feng Liu Qingjun Pan
}

Key Laboratory of Prevention and Management of Chronic Kidney Disease of Zhanjiang City, Institute of Nephrology, Affiliated Hospital of Guangdong Medical University, Zhanjiang, Guangdong, China

\section{Key Words}

Systemic lupus erythematosus • Th17 • Treg • Autophagy • Hydroxychloroquine (chloroquine)

\begin{abstract}
Background: Imbalanced cellular immunity is critical to the pathogenesis of systemic lupus erythematosus (SLE). Recently, autophagy has emerged as a key homeostatic mechanism in T lymphocytes. This study was conducted to explore the impact of autophagy on the Th17/ regulatory T (Treg) immune imbalance in SLE. Methods: Peripheral Th17 and Treg cells from newly diagnosed patients with SLE and healthy controls were detected by flow cytometry. Additionally, the effects of chloroquine (CQ) autophagic inhibition on the Th17/Treg immune response were investigated in vitro. In addition, hydroxychloroquine (HCQ) treatment of the Th17/Treg immune response and the disease progression of lupus MRL/lpr mice were studied in vivo. Results: Compared with healthy controls, both peripheral Th17 and Treg cells of patients with SLE exhibited activated autophagy, resulting in a heightened Th17 proinflammatory response and diminished Treg immunosuppression. Furthermore, in vitro experiments indicated that CQ autophagic inhibition effectively rebalanced the Th17/Treg immune responses in patients with SLE. In vivo studies of MRL/lpr mice similarly confirmed that HCQ treatment decisively inhibited the autophagy of Th17/Treg cellular subsets, restoring the immune balance, lowering the serum levels of inflammatory cytokines and autoantibodies, and improving renal histopathology. Conclusion: Activated autophagy contributed to the Th17/Treg immune imbalance in SLE, and chloroquine autophagic inhibition rebalanced Th17/ Treg-mediated immunity and ameliorated SLE.




\section{Cellular Physiology Cell Physiol Biochem 2017;44:412-422 \begin{tabular}{l|l|l} 
and BOI: 10.1159/000484955 & $\begin{array}{l}\text { (C) 2017 The Author(s). Published by S. Karger AG, Basel } \\
\text { www.karger.com/cpb }\end{array}$
\end{tabular}}

An et al.: CQ Autophagic Inhibition Rebalances Th17/Treg Response in SLE

\section{Introduction}

Systemic lupus erythematosus (SLE) is an autoimmune disease propagated by a host of inflammatory cytokines and autoantibodies, and it stems from imbalances in certain immune cell populations [1-3]. The impact of Th17/regulatory T (Treg) immune imbalances (i.e., elevated Th17 response, depressed Treg function) is critical to the pathogenesis of SLE [4-7], just like other autoimmune diseases [8]. Interleukin (IL)-17A, IL-17F, and IL-22 production by Th17 cells promotes the autoimmune response [9]. In conjunction with IL21 and B-cell activating factor (BAFF), overproduction of IL-17 enhances germinal center formation, encouraging $B$ cells to generate an abundance of pathogenic autoantibodies in SLE [10]. In contrast, Treg cells maintain immune homeostasis and suppress aberrant immune responses in a cytokine-dependent manner by releasing the immunosuppressive cytokines IL-10 and TGF- $\beta$. They also harbor a receptor protein, cytotoxic T-lymphocyteassociated protein 4 (CTLA-4), latently expressing TGF- $\beta$ on the membrane surface [11]. Although a large number of signaling pathways participate in the regulation of Treg cells [12-15], forkhead box protein 3 (Foxp3) as Treg specific transcription factor is the most important one. Diminished expression of Foxp3 in CD4 ${ }^{+} \mathrm{CD} 25^{+}$Treg cells created dysfunction of Treg cells in SLE [16]. Unfortunately, the mechanisms contributing to Th17/Treg immune imbalance in SLE remain unclear.

Recently, autophagy, an evolutionarily conserved cellular process that regulates the disassembly of unnecessary or dysfunctional cellular components in a lysosome-dependent manner [17], has emerged as a key homeostatic mechanism in T lymphocytes, affecting $\mathrm{T}$ cell-mediated immunity and autoimmunity [18-20]. In particular, autophagic deregulation in T lymphocytes from humans and mice with SLE was implicated in abnormal T-cell responses [21]. Consequently, we tested the hypothesis that autophagy might be pivotal in the imbalance of Th17/Treg immunity responsible for the progression of SLE, with autophagic regulation helping to rebalance Th17/Treg cellular responses. Moreover, chloroquine (CQ) is known as an autophagy inhibitor that impairs autophagic flux by blocking autophagic degradation [22], and hydroxychloroquine (HCQ), the hydroxylation of CQ has been widely used as a basic drug for SLE treatment [23].

This study aimed to investigate the role of CQ autophagic inhibition in the rebalance of Th17/Treg-mediated immunity in SLE based on studies of patients with SLE and spontaneous lupus-prone MRL-lpr/lpr mice.

\section{Materials and Methods}

\section{Clinical materials}

The study was conducted at the Affiliated Hospital of Guangdong Medical University (Zhanjiang, China), with approval from the Institutional Ethics Committee. All of the subjects, including newly diagnosed patients with SLE $(n=12)$ and healthy volunteer controls $(n=18)$, were recruited from the Department of Nephrology between December, 2014 and December, 2015, and all of them provided informed consent. The selected patients (male, 1; female, 11) were newly diagnosed with SLE, in accordance with modified SLE classification criteria formulated (1997) by the American College of Rheumatology (ACR). None had received glucocorticoids or other immunosuppressive agents, and they had no histories of hepatitis $B$, allergies, cancers, other severe systemic diseases, or other autoimmune disorders. Active disease was viewed as a SLE disease activity index (SLEDAI) score $\geq 10$. Blood samples were collected from each healthy volunteer (male, 3; female, 15). The two population subsets did not differ significantly in terms of sex or age.

\section{Animal models}

Female MRL/MpJ-Faslpr/J mice (age, 12 weeks old; weight, $33 \pm 2 \mathrm{~g}$ ) were supplied by the Shanghai SLAC Laboratory Animal Co., Ltd. (Shanghai, China). Also known as MRL/lpr mutants, these mice develop a lupus-like systemic autoimmune disease, marked by proliferation of aberrant $\mathrm{T}$ cells and hyperfunctional autoreactive B cells. The animals were housed in a special pathogen-free environment $\left(25^{\circ} \mathrm{C}\right.$, normal 12 - $\mathrm{h}$ light/12-h dark cycle) in the Animal Center of Guangdong Medical University. The mice had unrestricted 


\section{Cellular Physiology Cell Physiol Biochem 2017;44:412-422 \begin{tabular}{l|l} 
and Biochemistry Published online: November 14, 2017 & $\begin{array}{l}\text { C } 2017 \text { The Author(s). Published by S. Karger AG, Basel } \\
\text { www.karger.com/cpb }\end{array}$ \\
\hline DOI: 10.1159/00048455
\end{tabular}}

An et al.: CQ Autophagic Inhibition Rebalances Th17/Treg Response in SLE

access to standard food and sterilized water supplied by the facility. A total of 20 female MRL/lpr mice were equally and randomly assigned to hydroxychloroquine (HCQ) treatment or control groups. Each HCQtreated mouse received daily oral gavage of HCQ (100 mg/kg; Sanofi, Paris, France) diluted in saline (500 $\mu \mathrm{l}$ ), whereas the control mice were similarly administered equivalent volumes of saline only. All of the mice were euthanized after a 4-week treatment regimen, removing renal cortical tissue for histologic study. Blood samples were obtained by cardiac puncture of anaesthetized mice, separating the serum for enzymelinked immunosorbent assay (ELISA) and collecting peripheral blood mononuclear cells (PBMCs) for flow cytometry. All of the studies were approval by the Animal Experimentation Ethics Committee of Guangdong Medical University. Our experimental protocols conformed to approved guidelines.

\section{Flow cytometric analysis (FCM)}

Human and mouse blood samples were collected in anticoagulant-treated tubes. After centrifugation (to separate plasma), the supernatant was discarded, and the cell pellets were resuspended in red blood cell lysis buffer. PBMCs were isolated via Ficoll-Hypaque density-gradient centrifugation and were incubated ( 6 h) in complete RPMI 1640 medium with 10\% fetal bovine serum (Invitrogen, Carlsbad, CA, USA) with or without chloroquine (CQ) (10 $\mu \mathrm{mol} / \mathrm{L}$; Sigma-Aldrich Corp, St. Louis, MO, USA).

For cytokine analysis, PBMCs were incubated $(6 \mathrm{~h})$ in Cell Stimulation Cocktail (plus protein transport inhibitors) (Thermo Fisher Scientific, Waltham, MA, USA) prior to fixation and permeabilization. LC3-II detection (as described previously) [24] entailed the washing of PBMCs (0.1\% saponin in PBS) to extract the non-autophagosome-associated LC3-I before fixation.

After fixation and permeabilization, conjugated antibodies (direct or indirect) were applied to PBMCs for immunostaining, procuring the following antibodies and respective isotype controls (BioLegend, Inc., San Diego, CA, USA): anti-human CD4 (APC-eFlour780), anti-human IL-17A (PerCP-Cy 5.5), anti-human Foxp3 (eFlour660), anti-mouse CD4 (PE), anti-mouse/Rat IL-17A (PE-Cy7), and anti-mouse/Rat Foxp3 (APC). Rabbit anti-human LC3 (MBL International Corp, Woburn, MA, USA), rabbit anti-mouse LC3 (SigmaAldrich Corp), anti-human p62 (BD Biosciences, Franklin Lakes, NJ, USA) and respective isotype controls were obtained from other vendors. Fluorescein-labeled donkey anti-rabbit IgG (Alexa Fluor 488; Invitrogen) and the reagents for flow cytometry, including cell staining buffer, red blood cell lysis buffer (10×), fixation buffer, and permeabilization wash buffer $(10 \times)$ (BioLegend, Inc.) were also purchased. All of the commercial reagents were stored and used according to manufacturers' recommendations.

ELISA

ELISA kits were used to quantify levels of anti-nuclear antibody (ANA) and anti-dsDNA antibody (Alpha Diagnostic International, San Antonio, TX, USA) in mouse serum (1:100 dilution). Levels of IL-17, IFN- $\gamma$, and TGF- $\beta$ in human and mouse serum were also quantified via ELISA (Life Technologies Corp, Grand Island, NY, USA). All of the analyses were performed as instructed by the manufacturer.

\section{Western blotting}

Protein was extracted from human PBMCs, and western blot analysis was performed as previously detailed $[25,26]$. Primary antibodies to $\mathrm{p} 62$ protein and $\beta$-actin were purchased (Santa Cruz Biotechnology, Inc., Santa Cruz, CA, USA), as was anti-LC3B antibody (Sigma-Aldrich Corp). HRP-conjugated secondary antibodies were provided by the Beyotime Institute of Biotechnology (Nantong, Jiangsu, China).

\section{Statistical analysis}

All of the data are expressed as the mean \pm standard deviation (SD). To compare two groups, the independent sample $t$-test was applied. All of the computations relied on standard software (SPSS v16.0; SPSS Inc. [IBM], Chicago, IL, USA), setting significance at $p<0.05$.

\section{Results}

Demographics and clinical characteristics of patients with SLE

Various parameters of the 12 patients with SLE, each newly diagnosed, are presented in Table 1. 
Table 1. Demographic and clinical characteristics of patients with SLE $(\mathrm{n}=12)$. Abbreviations: ANA, anti-nuclear antibody; SLEDAI, systemic lupus erythematosus disease activity index; LN, lupus nephritis

\begin{tabular}{ll}
\hline Variables & Data \\
\hline Age (mean \pm SD, years) & $30 \pm 9.2$ \\
Gender (F/M, \%) & $11 / 1,91.7: 8.3$ \\
Disease duration (mean $\pm S D$, years) & $2.3 \pm 3.6$ \\
Anti-dsDNA Ab positive (n, \%) & $11,91.7$ \\
ANA positive (n, \%) & $11,91.7$ \\
Complement C3 decline (n, \%) & 12,100 \\
SLEDAI & $14.92 \pm 4.7$ \\
Mean $\pm S D$ & $14(10,28)$ \\
Median (minimum, maximum) & $10,83.4$ \\
LN & \\
Proteinuria (n, \%) & \\
\hline
\end{tabular}

Fig. 1. Patients with newly diagnosed SLE exhibit Th17/Treg imbalances in the peripheral blood. (A) Expressions levels of IL-17 (Th-17 cells) and Foxp3 (Treg cells) in patients with newly diagnosed SLE and in healthy controls, analyzed by flow cytometry; and (B-D) serum levels of IL-17, TGF- $\beta$, and IFN- $\gamma$ in patients with newly diagnosed SLE and in healthy controls by ELISA methods. Each bar, mean $\pm S D$; healthy controls $(\mathrm{n}=18)$; patients with newly diagnosed SLE $(\mathrm{n}=12) .{ }^{*} p<0.05$ and ${ }^{* * *} p<0.001$ vs. healthy controls.

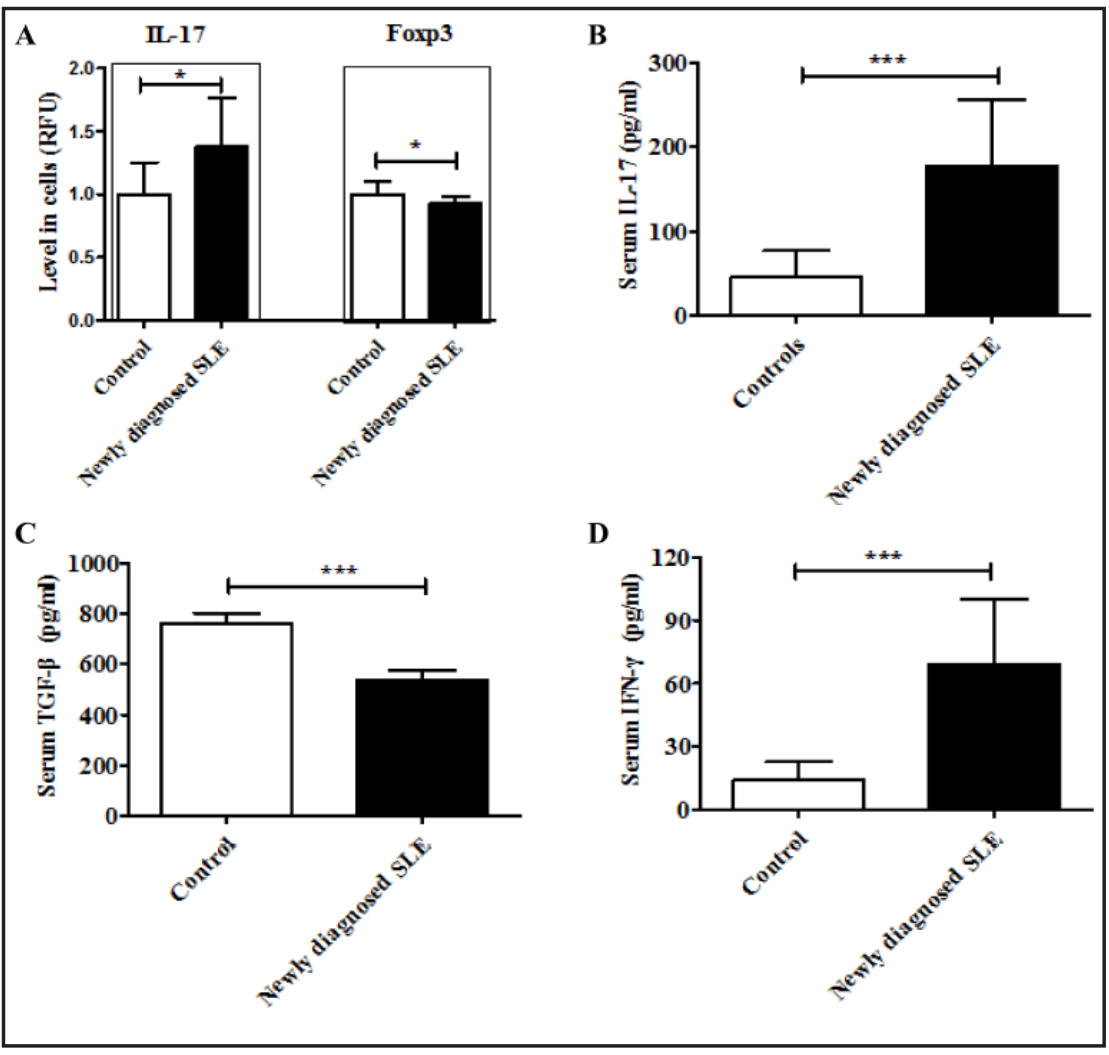

Patients with newly diagnosed SLE exhibit Th17/Treg imbalances, in conjunction with activated Th17/Treg autophagy

To first explore Th17/Treg immune responsiveness in SLE, we measured expression levels of IL-17 and Foxp3 in peripheral Th17 and Treg cells, respectively, using flow cytometry. As shown in Fig. 1A, patients with newly diagnosed SLE (vs. healthy controls) showed significantly greater IL-17 expression (in Th17 cells) but notably less Foxp3 expression (in Treg cells). We also measured IL-17, TGF- $\beta$, and IFN- $\gamma$ levels in serum to further investigate the imbalances of Th17/Treg cells in SLE. Relative to healthy controls, proinflammatory IL17 and IFN- $\gamma$ levels were markedly upregulated in patients with newly diagnosed SLE (Fig. $1 \mathrm{~B}$ and $1 \mathrm{D}$ ), whereas serum TGF- $\beta$ levels were significantly reduced in comparison (Fig. 1C). These data implicated Th17-cell overactivity and inhibition of Treg cells as immune derangements in patients with SLE.

To assess autophagic flux in immune cells from patients with SLE, we measured the expression of autophagic markers (LC3-II and p62) in peripheral PBMCs via western blotting, finding significantly greater LC3-II expression in cells from patients with newly diagnosed 
Fig. 2. Levels of autophagy-related proteins in PBMCs and in Th17/Treg cells from patients with newly diagnosed SLE. (A) Western blot analysis of LC3II and p62 expression in the PBMCs of patients with newly diagnosed SLE and healthy controls; and (B, C) LC3-II and p62 expression in Th17 and Treg cells from patients with newly diagnosed SLE and healthy controls, analyzed by flow cytometry. Each bar, mean \pm SD; healthy controls $(n=18)$; patients with newly diagnosed SLE $(\mathrm{n}=12)$. ${ }^{* *} p<0.01 v s$. healthy controls.

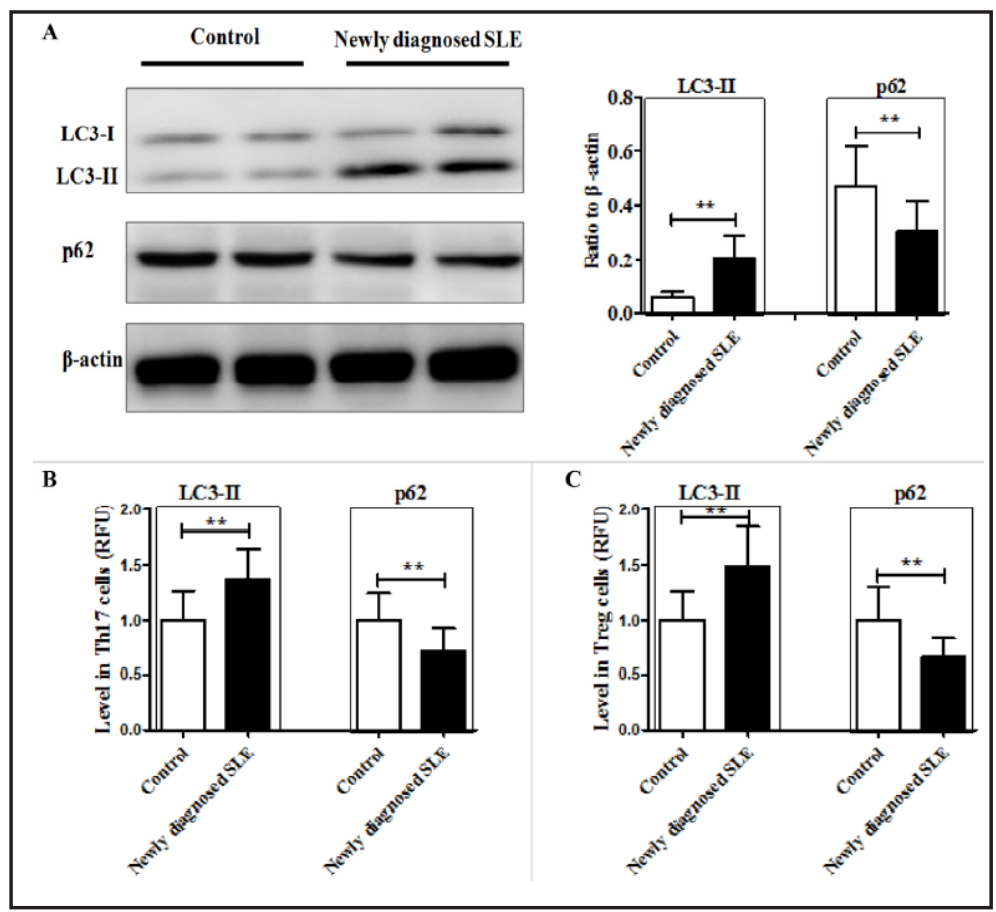

Fig. 3. In vitro $\mathrm{CQ}$ autophagic inhibition restores the balance between Th17 and Treg cells obtained from patients with SLE. (A) Western blot analysis of LC3-II and p62 expression levels in PBMCs from patients with newly diagnosed SLE in the presence or absence of in vitro CQ treatment; (B, C) LC3II and p62 expression levels in Th17 and Treg cells, analyzed by flow cytometry in patients with newly diagnosed SLE in the presence or absence of in vitro CQ treatment; and (D, E) IL17 and Foxp3 expression levels in Th17 cells, analyzed by flow cytometryin patients with newly diagnosed SLE in the presence or absence of in vitro CQ treatment. Each bar, mean \pm SD; patients with newly diagnosed SLE $(\mathrm{n}=12) .{ }^{*} p<0.05,{ }^{* *} p<0.01$ and $* * * p<0.001$ vs. untreated controls.

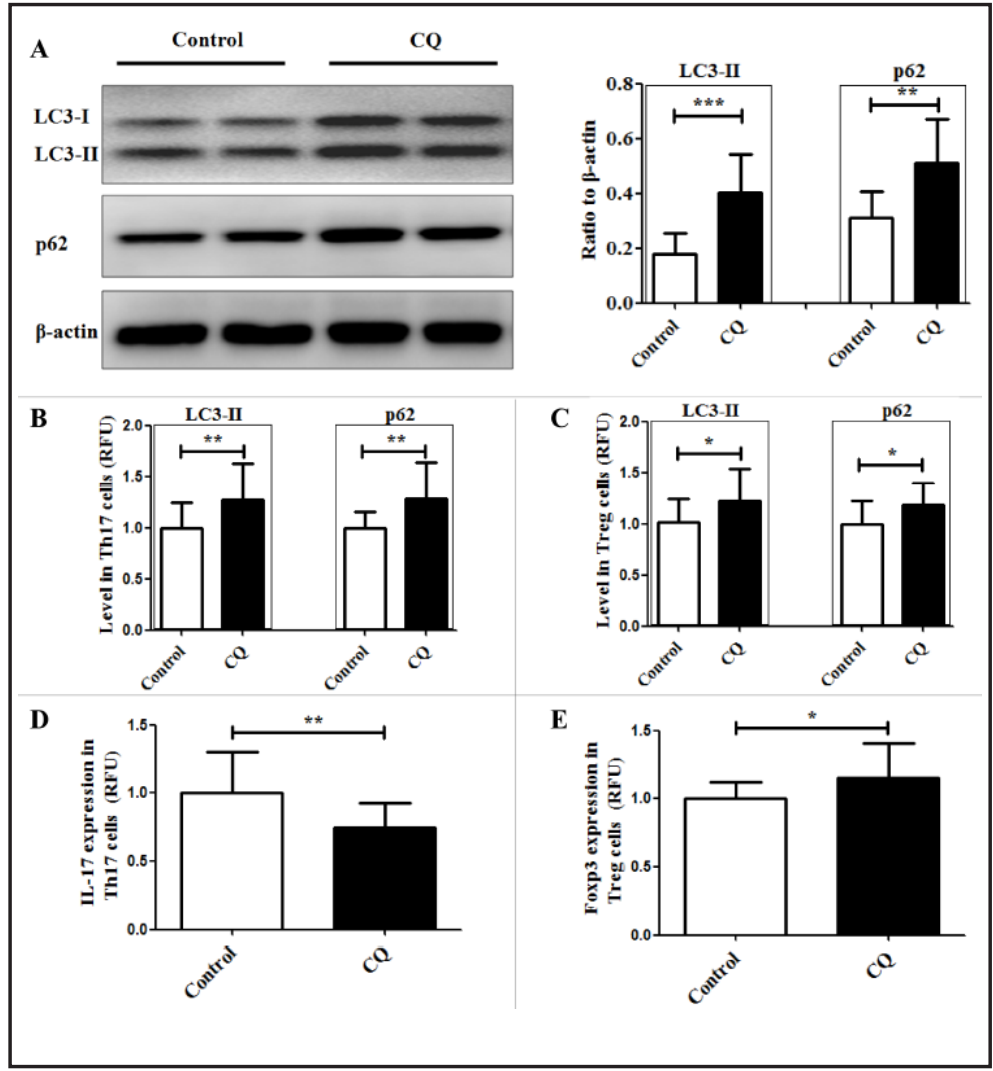

SLE, compared to healthy controls. We then determined whether LC3-II autophagic marker excess was due to autophagic activation or impaired autophagic degradation by examining the expression of p62, an autophagic substrate. Compared with controls, there was markedly less p62 in cells from patients with SLE (Fig. 2A). This combination of elevated LC3-II and diminished p62 indicated that autophagy was activated in PBMCs from patients with SLE. 
Fig. 4. In vivo HCQ autophagic inhibition rebalances the immune responses of peripheral Th17 and Treg cells in MRL/lpr mice. (A, B) LC3-II and p62 expression levels in Th17 and Treg cells from lupus-like mice in the presence or absence of HCQ treatment, analyzed by flow cytometry; and (C, D) IL-17 expression in Th17 cells and Foxp3 expression

A

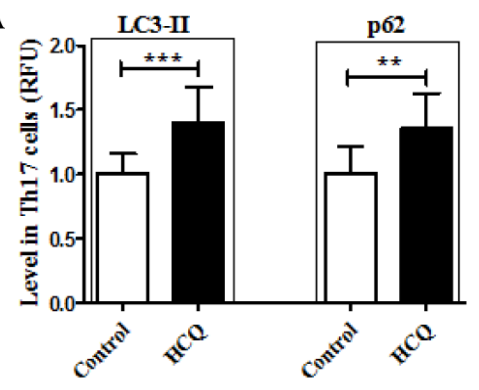

C

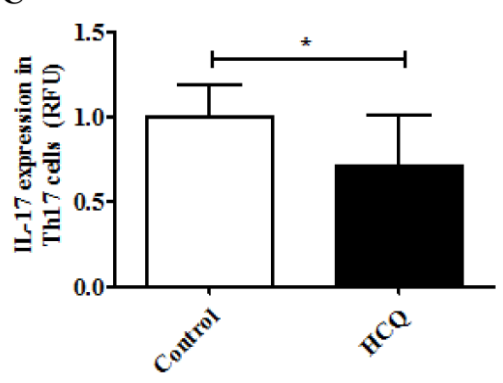

B

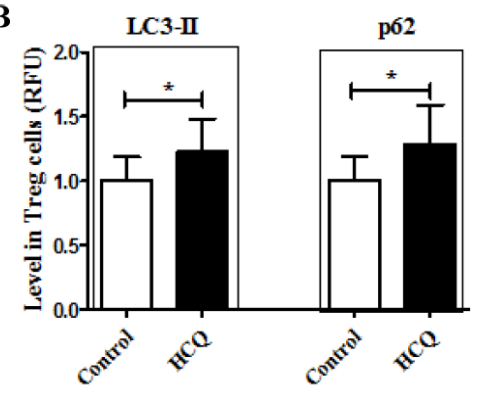

D

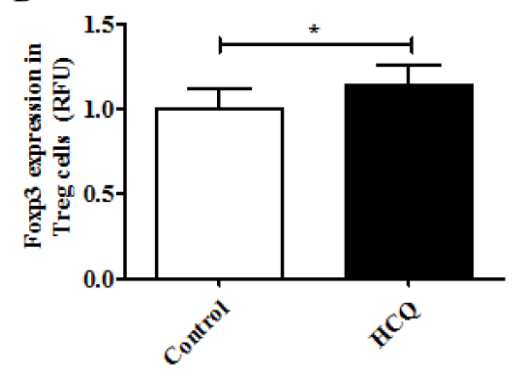

in Treg cells from lupus-like mice in the presence or absence of HCQ treatment, analyzed by flow cytometry. Each bar, mean $\pm \mathrm{SD}$; animal subsets $(\mathrm{n}=10) .{ }^{*} p<0.05,{ }^{* *} p<0.01$, and ${ }^{* * *} p<0.001$ vs. untreated controls.

Finally, we analyzed autophagy in Th17 and Treg cells by flow cytometry. Compared with healthy controls, LC3-II was markedly elevated and p62 diminished in both Th17 and Treg cells, thus implying activation of Th17/Treg autophagy. These results suggested that imbalanced Th17/Treg immune responses in patients with SLE were accompanied by Th17/ Treg autophagic activation.

In vitro CQ autophagic inhibition restores balance between Th17 and Treg cells obtained from patients with SLE

We further investigated the effects of autophagic inhibition on the immune responses of Th17 and Treg cells, using CQ as autophagic inhibitor in an in vitro experiment. CQ acts to disrupt the fusion of autophagosomes with lysosomes and to suppress lysosomal degradation by increasing lysosomal $\mathrm{pH}$ [27]. Through western blot analysis, we first assessed autophagic flux in PBMCs from patients with SLE after in vitro CQ exposure. According to our data, CQ (vs. control) treatment produced significant upregulation of LC3-II and p62 expression levels in PBMCs (Fig. 3A). Subsequently, these autophagic markers were analyzed in Th17 and Treg cells by flow cytometry. Again, levels of LC3-II and p62 were clearly elevated in both Th17 and Treg cells from patients with SLE after actual CQ exposure (Fig. 3B and 3C). As alluded to earlier, abundant p62, alongside LC3-II elevation, is a sign that autophagy in Th17 and Treg cells from patients with SLE patients was efficiently suppressed after CQ treatment. Interestingly, we also found that CQ (rather than control) treatment dramatically suppressed the expression of IL-17 in Th17 cells, with only a slight gain in Foxp3 expression by Treg cells from patients with SLE (Fig. 3D and 3E). This in vitro evidence suggested that autophagic inhibition by CQ could rebalance the immune responses of Th17 and Treg cells in patients with SLE.

In vivo HCQ autophagic inhibition rebalances immune responses of peripheral Th17 and Treg cells in MRL/lpr mice

To ascertain the role of autophagic inhibition in immune responses mediated by Th17 and Treg cells, we investigated the in vivo effects of HCQ (an autophagic inhibitory agent) 
Fig. 5. HCQ autophagic inhibition suppresses autoimmune responses and alleviates pathologic changes in the kidneys in MRL/lpr mice. (A) Serum levels of IL17 , TGF- $\beta$, and IFN- $\gamma$ determined by ELISA in lupus-like mice, with or without HCQ treatment; (B) serum anti-dsDNA IgG and anti-nuclear IgG determinations by ELISA in lupus-like mice, with or without HCQ treatment; and (C) representative photomicrographs of

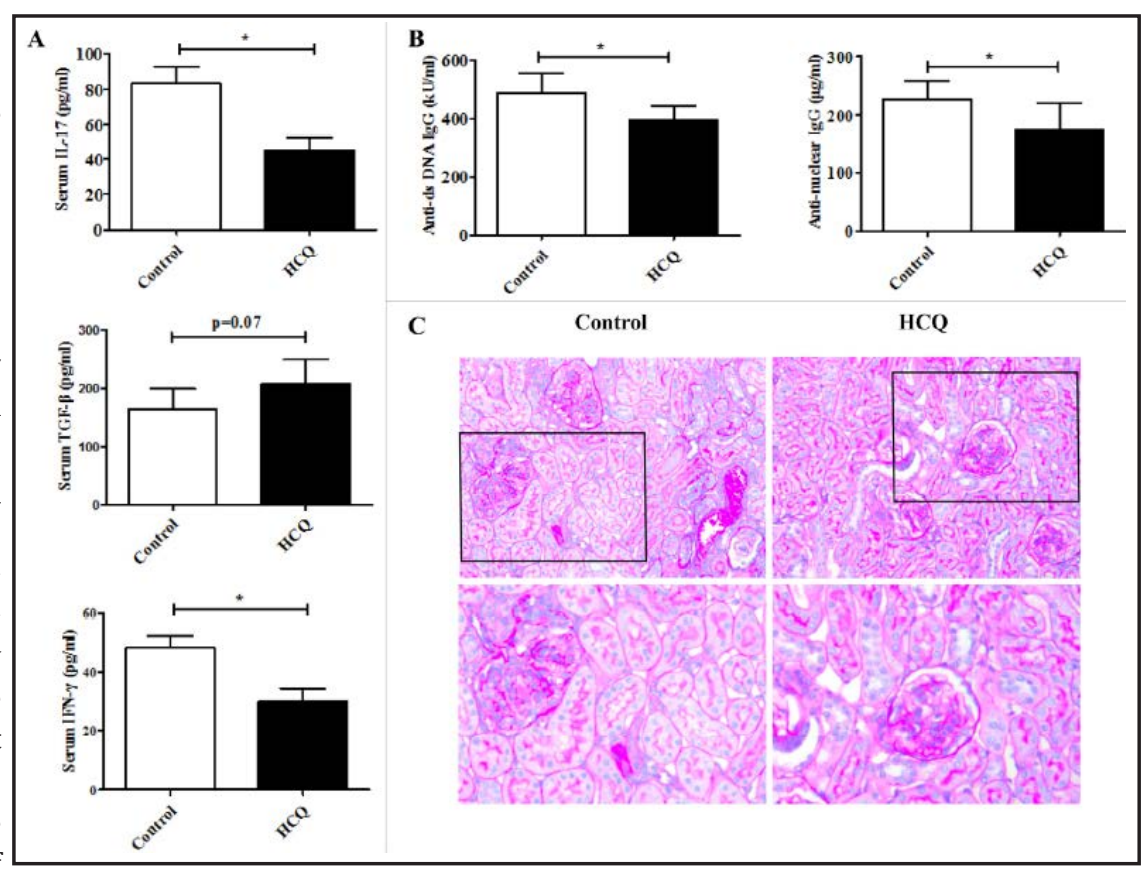
PAS-stained renal tissue from lupus-like mice, with or without HCQ treatment (upper, $\times 200$; lower, $\times 400$ ). Each bar, mean \pm SD; animal subsets $(\mathrm{n}=10) .{ }^{*} p<0.05$ vs. untreated controls.

on Th17/Treg imbalances in lupus-like MRL/lpr mice. As shown in Fig. 4A and 4B, HCQ treatment led to significantly greater expression levels of LC3-II and p62 in peripheral Th17 and Treg cells from MRL/lpr mice (compared with controls), with significantly less IL-17 expression in Th17 cells and higher levels of Foxp3 in Treg cells by comparison (Fig. 4C and 4D). These data were compatible with the restoration of immune responses mediated by Th17 and Treg cells in lupus-like mice.

HCQ autophagic inhibition suppresses autoimmune responses and alleviates pathologic changes in kidneys of MRL/lpr mice

Finally, we examined the effects of HCQ treatment on autoimmune responses in lupuslike mice, using ELISA to assess serum levels of IL- 17 and IFN- $\gamma$ as proinflammatory cytokines, anti-dsDNA IgG, and anti-nuclear IgG, as well as the immunosuppressive cytokine TGF- $\beta$. HCQ treatment of MRL/lpr mice resulted in significantly reduced serum concentrations of IL-17, IFN- $\gamma$, anti-dsDNA IgG, and anti-nuclear IgG (contrasting greatly with untreated controls), with a marginal increase in TGF- $\beta$ (Fig. 5A and 5B). As shown in Fig. 5C, HCQ treatment also remarkably attenuated expected kidney changes, inhibiting mesangial cellular proliferation and suppressing renal tubular and interstitial infiltration by mononuclear/lymphocytic cells, in a clear departure from the controls. Thus, HCQ autophagic inhibition was capable of modulating aberrant immune responses and attenuating kidney injury in lupus-like mice.

\section{Discussion}

Herein, we substantiated the importance of autophagic activation as a critical accompaniment of the Th17/Treg imbalances that fuel the progression of SLE. Indeed, inhibition of autophagy by CQ or HCQ serves to dampen Th17 cellular responses and to promote the immunosuppressive effects of Treg cells, thus reducing proinflammatory cytokine levels and anti-dsDNA antibody production.

New information emerging about the balance between Th17 and Treg cells has offered a new paradigm for the immune underpinnings that control the onset and progression of SLE 


\section{Cellular Physiology Cell Physiol Biochem 2017;44:412-422 \begin{tabular}{l|l|l} 
DOI: 10.1159/000484955 & O 2017 The Author(s). Published by S. Karger AG, Basel \\
wwww.karger.com/cpb
\end{tabular} \\ An et al.: CQ Autophagic Inhibition Rebalances Th17/Treg Response in SLE}

$[4,5]$. In the present investigation, we determined that patients newly diagnosed with SLE patients (and therefore unexposed to immunosuppressive treatment) displayed increases in peripheral Th17 cells, with elevated levels of serum IL-17, IFN- $\gamma$ and anti-dsDNA antibodies but diminished circulating Treg cells and serum levels of TGF- $\beta$, an immunosuppressive cytokine. Our findings indicated that Th17/Treg immune responses were indeed imbalanced in such patients, corresponding to data from human [6, 28] and experimental models [7] previously reported by other researchers. However, the driving force of Th17/Treg imbalance remains unclear [29].

Recently, autophagy has been implicated in T-lymphocyte homeostasis and in T cellpredominant autoimmune diseases, including SLE [30]. Autophagy is a degradative process whereby cytoplasmic content is engulfed by lysosomes and destroyed [31]. It is well known that microtubule-associated protein 1 light chain 3 (LC3)-II and p62 are key markers used to monitor autophagic flux [26, 32]. An increase in LC3-II indicates a buildup of autophagic vacuoles, reflecting either increased formation or decreased clearance. In contrast, p62 is a ubiquitin-binding autophagic receptor and signaling protein, the accumulation of which denotes autophagy-lysosomal pathway disruption, culminating in aggregates of autophagic vacuoles [33]. Our data confirmed increased LC3 and diminished p62 expression levels in PBMCs from patients with SLE, indicating excessive autophagic activation, consistent with previous reports contending for a pivotal role of autophagy in the abnormal activation of $\mathrm{T}$ cells in SLE sufferers, all supported by genetic, cell-biology, and model-animal studies $[21,34,35]$. Hence, we presumed that autophagy might be critical in Th17/Treg immune imbalances due to progressive SLE.

To test this hypothesis, our first step was to assess autophagic activity in Th17 and Treg cells collected from patients with newly diagnosed SLE and healthy controls. We subsequently observed remarkable upregulation of LC3-II expression and diminished p62 expression in Th17 and Treg cells from these patients with SLE, based on flow cytometric analysis. One previous study postulated a constitutively higher level of autophagy in T-cell populations of patients with SLE [21], but no other publications have addressed autophagy in T-cell subsets, not even Th17 and Treg cells. Nonetheless, our data confirmed autophagic activation in Th17 and Treg cells from patients with SLE, in conjunction with Th17/Treg immune imbalance.

Fig. 6. Schematic representation of the effect of CQ on Th17/Treg immune imbalance via inhibiting autophagy in SLE. Both Th17 and Treg cells in SLE exhibit activated autophagy, and blocking of the autophagy pathway by CQ could rebalance the Th17/Treg response in SLE.

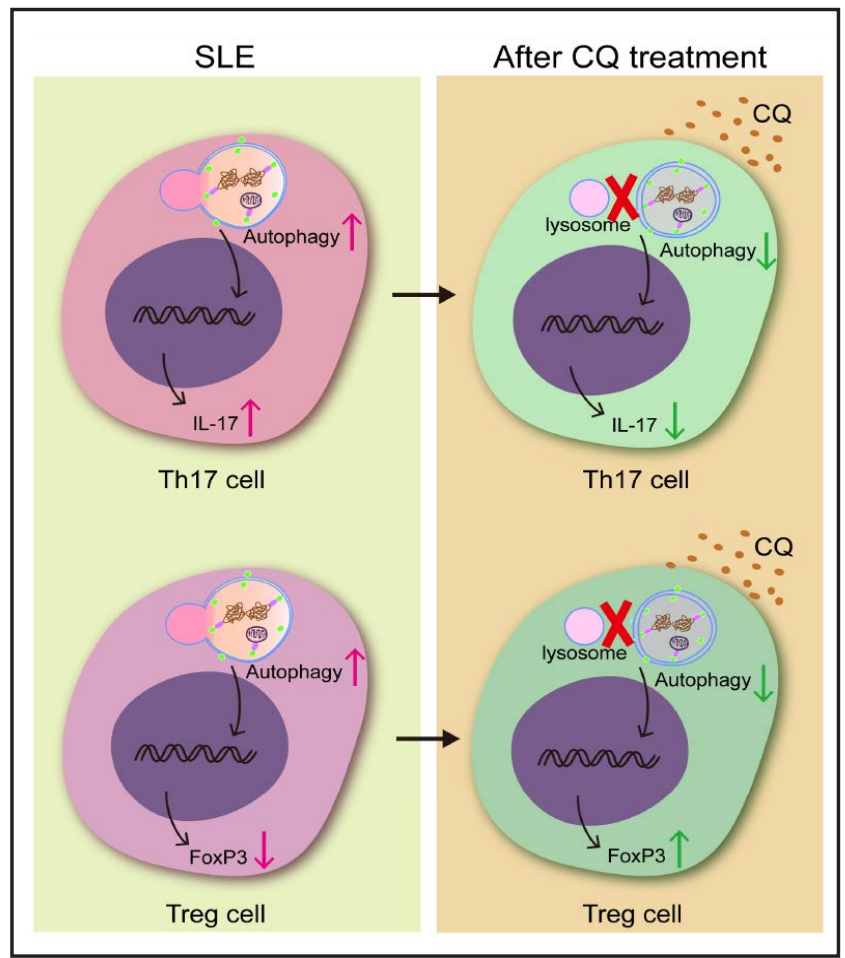




\section{Cellular Physiology Cell Physiol Biochem 2017;44:412-422 \begin{tabular}{ll|l} 
DOI: 10.1159/000484955 & O 2017 The Author(s). Published by S. Karger AG, Basel \\
wwww.karger.com/cpb
\end{tabular}

To then clarify the role of autophagic activation in Th17/Treg cells, we blocked the autophagic pathway, using CQ to inhibit autophagic-lysosomal degradation in vitro. As expected, CQ markedly enhanced LC3-II and p62 expression levels in both Th17 and Treg cells obtained from patients with SLE. At the same time, IL-17 expression in Th17 cells was significantly suppressed, with a slight upturn in Foxp3 expression by Treg cells. These outcomes were further affirmed in HCQ-treated MRL/lpr lupus-like mice, in which decisive upregulation of LC3-II and p62 expression in peripheral Th17 and Treg cells, a significant decrease in IL-17 expression by Th17 cells, and upregulation of Foxp3 expression in Treg cells were all evident. Additionally, HCQ treatment substantially reduced serum levels of IL-17 and IFN- $\gamma$, suppressing autoantibody production and alleviating kidney damage in these animals. Thus, the use of autophagic-lysosomal inhibitors to downregulate excessive autophagic activation could potentially correct the perceived imbalances of Th17/Treg cells in SLE and attenuate immune manifestations.

Notably, we found that downregulation of autophagy in both Th17 and Treg cells of patients with SLE dampened Th17 immune responses while also promoting Treg immunosuppressive effects. Therefore, it seems that the effects of autophagy on diverse T-cell subsets might actually differ. Similarly, inhibition of autophagy reduces IL-17 secretion by reversing the overactivity of Th17 cells observed in SLE, whereas Foxp3 in Treg cells increases during the course of autophagic inhibition (Fig. 6). A potential explanation is that autophagic-lysosomal inhibitors could block lysosome-dependent degradation of Foxp3, which is crucial for Treg cellular differentiation or function. Clearly, CQ does prevent lysosomedependent degradation of Foxp3, preserving Treg-cell functionality [36]. This phenomenon was similarly described in an earlier report of reduced severity of experimental autoimmune encephalomyelitis, achieved through CQ-induced enhancement of Treg functionality [37]. However, the means by which such differential autophagic regulation occurs will require further research. It might depend upon metabolic factors, with degradation of specific proteins mediated by the autophagic process.

In this study, CQ (HCQ) was employed as an autophagic inhibitor to downregulate autophagy but not by RNA interference due to safety concerns of RNAi delivery with viral vectors or with nonviral delivery methods with the potential to activate Th17/Treg cells from SLE patients. In another aspect, autophagy-knockout lupus mice were still not obtained.

In conclusion, activated autophagy contributed to Th17/Treg immune imbalance in SLE, and CQ (HCQ) autophagic inhibition rebalanced Th17/Treg-mediated immunity and ameliorated SLE.

\section{Acknowledgements}

National Natural Science Foundation of China (81470959, 81202346, 81471530), Science and Technology Planning Project of Zhanjiang City, China (2016B01099), Medical Scientific Research Foundation of Guangdong Province, China (A2016519) and Special Funds for the Cultivation of Guangdong College Students' Scientific and Technological Innovation (“Climbing Program" Special Funds).(NO. pdjh2017b0228).

\section{Disclosure Statement}

None.

\section{References}

1 Crispin JC, Kyttaris VC, Terhorst C, Tsokos GC: T cells as therapeutic targets in SLE. Nat Rev Rheumatol 2010;6:317-325.

2 Sanz I, Lee FE: B cells as therapeutic targets in SLE. Nat Rev Rheumatol 2010;6:326-337. 


\section{Cellular Physiology Cell Physiol Biochem 2017;44:412-422 \begin{tabular}{c|c|c|c|} 
DOI: 10.1159/000484955 & O 2017 The Author(s). Published by S. Karger AG, Basel \\
www.karger.com/cpb
\end{tabular}

3 Ramanujam M, Davidson A: Targeting of the immune system in systemic lupus erythematosus. Expert Rev Mol Med 2008;10:e2.

4 Yang J, Yang X, Zou H, Chu Y, Li M: Recovery of the immune balance between Th17 and regulatory T cells as a treatment for systemic lupus erythematosus. Rheumatology (Oxford) 2011;50:1366-1372.

5 Alunno A, Bartoloni E, Bistoni O, Nocentini G, Ronchetti S, Caterbi S, Valentini V, Riccardi C, Gerli R: Balance between regulatory $\mathrm{T}$ and Th17 cells in systemic lupus erythematosus: the old and the new. Clin Dev Immunol 2012;2012:823085.

-6 Xing Q Wang B, Su H, Cui J, Li J: Elevated Th17 cells are accompanied by FoxP3+ Treg cells decrease in patients with lupus nephritis. Rheumatol Int 2012;32:949-958.

7 Yang J, Chu Y, Yang X, Gao D, Zhu L, Yang X, Wan L, Li M: Th17 and natural Treg cell population dynamics in systemic lupus erythematosus. Arthritis Rheum 2009;60:1472-1483.

-8 Li C, Yuan J, Zhu YF, Yang XJ, Wang Q, Xu J, He ST, Zhang JA: Imbalance of Th17/Treg in Different Subtypes of Autoimmune Thyroid Diseases. Cell Physiol Biochem 2016;40:245-252.

-9 Patel DD, Kuchroo VK: Th17 Cell Pathway in Human Immunity: Lessons from Genetics and Therapeutic Interventions. Immunity 2015;43:1040-1051.

10 Hsu HC, Yang P, Wang J, Wu Q, Myers R, Chen J, Yi J, Guentert T, Tousson A, Stanus AL, Le TV, Lorenz RG, Xu H, Kolls JK, Carter RH, Chaplin DD, Williams RW, Mountz JD: Interleukin 17-producing T helper cells and interleukin 17 orchestrate autoreactive germinal center development in autoimmune BXD2 mice. Nat Immunol 2008;9:166-175.

11 Josefowicz SZ, Lu LF, Rudensky AY: Regulatory T cells: mechanisms of differentiation and function. Annu Rev Immunol 2012;30:531-564.

12 Zhou Y, Salker MS, Walker B, Munzer P, Borst O, Gawaz M, Gulbins E, Singh Y, Lang F: Acid Sphingomyelinase (ASM) is a Negative Regulator of Regulatory T Cell (Treg) Development. Cell Physiol Biochem 2016;39:985995.

13 Ding J, Su J, Zhang L, Ma J: Crocetin Activates Foxp3 Through TIPE2 in Asthma-Associated Treg Cells. Cell Physiol Biochem 2015;37:2425-2433.

14 Li S, Fan Q, He S, Tang T, Liao Y, Xie J: MicroRNA-21 negatively regulates Treg cells through a TGF-beta1/ Smad-independent pathway in patients with coronary heart disease. Cell Physiol Biochem 2015;37:866878.

15 Shen Y, Wei Y, Wang Z, Jing Y, He H, Yuan J, Li R, Zhao Q, Wei L, Yang T, Lu J: TGF-beta regulates hepatocellular carcinoma progression by inducing Treg cell polarization. Cell Physiol Biochem 2015;35:1623-1632.

16 Valencia X, Yarboro C, Illei G, Lipsky PE: Deficient CD4+CD25high T regulatory cell function in patients with active systemic lupus erythematosus. J Immunol 2007;178:2579-2588.

17 Shibutani ST, Saitoh T, Nowag H, Munz C, Yoshimori T: Autophagy and autophagy-related proteins in the immune system. Nat Immunol 2015;16:1014-1024.

$>18$ Bronietzki AW, Schuster M, Schmitz I: Autophagy in T-cell development, activation and differentiation. Immunol Cell Biol 2015;93:25-34.

19 Ma Y, Galluzzi L, Zitvogel L, Kroemer G: Autophagy and cellular immune responses. Immunity 2013;39:211227.

20 Liu G, Bi Y, Wang R, Wang X: Self-eating and self-defense: autophagy controls innate immunity and adaptive immunity. J Leukoc Biol 2013;93:511-519.

21 Alessandri C, Barbati C, Vacirca D, Piscopo P, Confaloni A, Sanchez M, Maselli A, Colasanti T, Conti F, Truglia S, Perl A, Valesini G, Malorni W, Ortona E, Pierdominici M: T lymphocytes from patients with systemic lupus erythematosus are resistant to induction of autophagy. FASEB J 2012;26:4722-4732.

22 Mizushima N, Yoshimori T, Levine B: Methods in mammalian autophagy research. Cell 2010;140:313-326.

23 Bertsias GK, Tektonidou M, Amoura Z, Aringer M, Bajema I, Berden JH, Boletis J, Cervera R, Dorner T, Doria A, Ferrario F, Floege J, Houssiau FA, Ioannidis JP, Isenberg DA, Kallenberg CG, Lightstone L, Marks SD, Martini A, Moroni G, Neumann I, Praga M, Schneider M, Starra A, Tesar V, Vasconcelos C, van Vollenhoven RF, Zakharova H, Haubitz M, Gordon C, Jayne D, Boumpas DT, European League Against R, European League Against Rheumatism and European Renal Association-European Dialysis and Transplant Association: Joint European League Against Rheumatism and European Renal Association-European Dialysis and Transplant Association (EULAR/ERA-EDTA) recommendations for the management of adult and paediatric lupus nephritis. Ann Rheum Dis 2012;71:1771-1782. 


\section{Cellular Physiology Cell Physiol Biochem 2017;44:412-422 \begin{tabular}{ll|l} 
DOI: 10.1159/000484955 & $\begin{array}{l}\text { O 2017 The Author(s). Published by S. Karger AG, Basel } \\
\text { www.karger.com/cpb }\end{array}$
\end{tabular}}

An et al.: CQ Autophagic Inhibition Rebalances Th17/Treg Response in SLE

24 Eng KE, Panas MD, Karlsson Hedestam GB, McInerney GM: A novel quantitative flow cytometry-based assay for autophagy. Autophagy 2010;6:634-641.

25 Liu WJ, Luo MN, Tan J, Chen W, Huang LZ, Yang C, Pan Q Li B, Liu HF: Autophagy activation reduces renal tubular injury induced by urinary proteins. Autophagy 2014;10:243-256.

26 Liu WJ, Shen TT, Chen RH, Wu HL, Wang YJ, Deng JK, Chen QH, Pan Q, Huang Fu CM, Tao JL, Liang D, Liu HF: Autophagy-Lysosome Pathway in Renal Tubular Epithelial Cells Is Disrupted by Advanced Glycation End Products in Diabetic Nephropathy. J Biol Chem 2015;290:20499-20510.

27 Cheong H, Lu C, Lindsten T, Thompson CB: Therapeutic targets in cancer cell metabolism and autophagy. Nat Biotechnol 2012;30:671-678.

-28 Talaat RM, Mohamed SF, Bassyouni IH, Raouf AA: Th1/Th2/Th17/Treg cytokine imbalance in systemic lupus erythematosus (SLE) patients: Correlation with disease activity. Cytokine 2015;72:146-153.

29 Hall BM: T Cells: Soldiers and Spies--The Surveillance and Control of Effector T Cells by Regulatory T Cells. Clin J Am Soc Nephrol 2015;10:2050-2064.

-30 Oral O, Yedier O, Kilic S, Gozuacik D: Involvement of autophagy in T cell biology. Histol Histopathol 2017;32:11-20.

31 Yang Z, Klionsky DJ: Mammalian autophagy: core molecular machinery and signaling regulation. Curr Opin Cell Biol 2010;22:124-131.

-32 Liu WJ, Xu BH, Ye L, Liang D, Wu HL, Zheng YY, Deng JK, Li B, Liu HF: Urinary proteins induce lysosomal membrane permeabilization and lysosomal dysfunction in renal tubular epithelial cells. Am J Physiol Renal Physiol 2015;308:F639-649.

-33 Johansen T, Lamark T: Selective autophagy mediated by autophagic adapter proteins. Autophagy 2011;7:279-296.

34 Pan Q Gao C, Chen Y, Feng Y, Liu WJ, Liu HF: Update on the role of autophagy in systemic lupus erythematosus: A novel therapeutic target. Biomed Pharmacother 2015;71:190-193.

35 Pierdominici M, Vomero M, Barbati C, Colasanti T, Maselli A, Vacirca D, Giovannetti A, Malorni W, Ortona E: Role of autophagy in immunity and autoimmunity, with a special focus on systemic lupus erythematosus. FASEB J 2012;26:1400-1412.

36 Du T, Nagai Y, Xiao Y, Greene MI, Zhang H: Lysosome-dependent p300/FOXP3 degradation and limits Treg cell functions and enhances targeted therapy against cancers. Exp Mol Pathol 2013;95:38-45.

-37 Thome R, Moraes AS, Bombeiro AL, Farias Ados S, Francelin C, da Costa TA, Di Gangi R, dos Santos LM, de Oliveira AL, Verinaud L: Chloroquine treatment enhances regulatory T cells and reduces the severity of experimental autoimmune encephalomyelitis. PLoS One 2013;8:e65913. 